\title{
STUDY ON ALLELOPATHY OF THREE SPECIES OF PINUS IN NORTH CHINA
}

\author{
ZHANG, Z. X. ${ }^{1,2}-$ GAO, Y. ${ }^{1,3^{*}}-$ ZHAO, Y. J. ${ }^{4}$ \\ ${ }^{1}$ Shandong Provincial Key Laboratory of Water and Soil Conservation and Environmental \\ Protection, College of Resources and Environment, Linyi University, 276005 Linyi, China \\ ${ }^{2}$ Linyi No. 4 High School of Shandong Province, 276000 Linyi, China \\ (e-mail:1431873619@qq.com) \\ ${ }^{3}$ Linyi Scientific Exploration Laboratory, 276037 Linyi, China \\ ${ }^{4}$ College of Agriculture and Forestry, Linyi University, 276005 Linyi, China \\ (e-mail: zhaoyanjie1882@126.com) \\ *Corresponding author \\ e-mail: gaoyuan1182@tom.com,gaoy@lyu.edu.cn \\ (Received $31^{\text {st }}$ May 2018; accepted $13^{\text {th }}$ Sep 2018)
}

\begin{abstract}
Two-year-old seedlings of Pinus species typical in North China (Pinus thunbergii, Pinus tabuliformis and Pinus koraiensis) were selected as the research object. The water extracted from the litter of $P$. thunbergii, $P$. tabuliformis and $P$. koraiensis were used to test the changes of photosynthetic pigment Chl. a, Chl. b and allelochemicals. The results were as follows: aqueous extract of $P$. thunbergii needle litter had significantly low inhibition on $P$. thunbergii while strongly inhibited $P$. tabuliformis and $P$. koraiensis. It also showed a markedly low inhibition on $P$. thunbergii, a moderate inhibition on $P$. tabuliformis, and a greatly strong inhibition on $P$. koraiensis. Obviously strong inhibition on $P$. thunbergii, P. tabuliformis and $P$. koraiensis were observed as well. Our study showed that pine needle water leaching solutions, such as $P$. tabuliformis, $P$. thunbergii and $P$. koraiensis, significantly inhibit their Chl. a and Chl. b, and will be a norm, which provides a new explanation for the decline in the coniferous forests of northern China.
\end{abstract}

Keywords: Pinus thunbergii, Pinus tabuliformis, Pinus koraiensis, allelopathy, Chl. a, Chl. b

\section{Introduction}

Allelopathy refers to the process that those chemical substances produced by plants, which can have beneficial or detrimental effects on the growth of the plants themselves or other plants around, are released into the environment through leaching, volatilization, stubble degradation and root excretion, (Peng and Shao, 2001; Kong et al., 2002; Zhou et al., 2004). Studies on allelopathy have dramatically increased since the 1970s and have undergone rapid development since the 1990s. In recent years, the research has become a hot topic in the fields of botany, ecology, agronomy and soil science (Albuquerque et al., 2011; Cheng and Cheng, 2015). At present, allelochemicals mean basically secondary metabolites. The most common allelochemicals are organic acids, phenols and terpenoids (Yan et al., 2000; Chen et al., 2015).

Allelopathy exists widely in nature and significantly affects forest community succession, vegetation restoration and forestry production (Li et al., 2010; Albuquerque et al., 2011; Cheng and Cheng, 2015). Conifers are important timber and afforestation species in China. In recent years, many coniferous plantations have faced such problems as productivity decline, soil degradation and natural regeneration obstacles. The traditional view is that this phenomenon is caused by rotation, inappropriate harvesting 
methods, harvesting residues, tillage and land preparation (Chen et al., 2003; Wang et al., 2007). However, in recent years, convincing evidence has showed that allelopathy may be the key factor affecting the natural regeneration of coniferous forests. Allelochemicals can be transported to the ground through pine needles and litters (Kimura et al., 2015), which will limit the growth of seedlings and herbaceous plants (Kil and Yang, 1983; KatoNoguchi et al., 2011) and will impede the regeneration of the stands (Pan et al., 2009; Wang et al., 2007). Researches on the allelopathy of the main afforestation species in northern China can provide important theoretical and practical guidance for the management and survival of regional man-made forests.

The type and dose of allelochemicals released by plants to the environment are decided by combined effects, namely the plant itself and environmental factors. Environmental stress can increase the release of allelochemicals (Albuquerque et al., 2011). Therefore, both the environment and the plant itself must be taken into consideration in the study of allelopathy. $P$. thunbergii, $P$. tabuliformis and $P$. koraiensis are the main afforestation species in northern China (Wei et al., 2017; Yin et al., 2018). At present, only few studies focus on the allelopathic effects of $P$. thunbergii tissue and understory extracts on Brassica rapa pekinensis (Zhang et al., 2012), litter leaf and soil extract on seed germination and seedling growth of $P$. koraiensis (Chen et al., 2016), Allelopathic effects on the growth and photosynthesis of P. tabuliformis seedlings (Jia et al., 2003), the autotoxicity of $P$. tabuliformis ( $\mathrm{Li}$ et al., 2010), the allelopathic effect of $P$. tabuliformis soil in the Loess Plateau (Zhu et al., 2014), and the allelopathic effect of P. tabuliformis root in Hilly Loess Plateau (Wang et al., 2015), etc. There is no study focusing on the interaction between $P$. thunbergii, $P$. tabuliformis and P. koraiensis. In this research, 2year-old seedlings of $P$. thunbergii, $P$. tabuliformis and $P$. koraiensis were selected for the pot experiment to study the effects of water and aqueous solution of $P$. thunbergii pine needles, $P$. tabuliformis pine needles and $P$. koraiensis pine needles. Changes of Chl. a, Ch. b, allelochemicals, also total triterpenes in seedlings were examined after 30 days.

\section{Materials and methods}

\section{Testing materials}

The pine needle litter of $P$. thunbergii and $P$. tabuliformis was collected from Tianmeng area of Mountain Meng, China. All target trees grew in a natural environment, with a height of about $10 \mathrm{~m}$, and a diameter of about $15 \mathrm{~cm}$. The pine needle litter of $P$. koraiensis was purchased from Hunchun seedling breeding base in Jilin, China. $P$. thunbergii and P. tabuliformis seedlings were purchased from the seedling breeding base of Junan County in Linyi city, China, the seedlings were about $0.5 \mathrm{~m}$ in height and $1.5 \mathrm{~cm}$ in diameter. $P$. koraiensis seedlings were purchased from Hunchun seedling breeding base in Jilin, China, the seedlings were about $0.5 \mathrm{~m}$ in height and $1.5 \mathrm{~cm}$ in diameter. The pot experiment was conducted at the science exploration laboratory base in Linyi, China.

\section{Testing method}

1) Five $600 \mathrm{~m}^{2}$ P. thunbergii and P. tabuliformis sample plots were set up, and a $1 \mathrm{~m}^{2}$ litter box was placed in each plot; 2) From January 2017 to July 2017, pine needles in the collection boxes of $P$. thunbergii and $P$. tabuliformis were packaged and transferred to the laboratory and summarized for natural drying; 3) From July 25 to August 25, 2017, pine needle litters of $P$. thunbergii, $P$. tabuliformis and $P$. koraiensis were soaked; 4) From July 
20 to August 25, 2017, P. thunbergii, $P$. tabuliformis and $P$. koraiensis seedlings were potted; 5) From August 25 to September 25, 2017, the seedlings of $P$. thunbergii, $P$. tabuliformis and $P$. koraiensis were inoculated in pots. Four treatments (clean water control group, $P$. thunbergii needle water leaching solution treatment group, $P$. tabuliformis needle water leaching solution treatment group and $P$. koraiensis needle water leaching solution treatment group) were set for each of the three young pine trees. Five plants were cultivated in parallel. $100 \mathrm{~mL}$ water or pine needles water leachate was added to the young pine trees every 5 days for 6 times, a total volume of $600 \mathrm{~mL}$ was used; 5) On September 25, all pine needles were sampled and sealed in bags for inspection.

\section{Determination method}

\section{Determination of Chl. $a$ and Chl. b content}

$0.1 \mathrm{~g}$ pine needles were weighed, cut and placed into the mortar. More than $15 \mathrm{~mL}$ absolute ethanol was added to the mortar until the total volume of the liquid was $25 \mathrm{~mL}$. These solutions were stored in the dark for $24 \mathrm{~h}$ and then were filtered. The pigment's optical density was measured by a spectrophotometer 721 at a wavelength of $665 \mathrm{~nm}$ and $649 \mathrm{~nm}$, and the content Chl. a and Chl. b were calculated.

\section{Determination of total triterpene content}

After extraction and centrifugation of the pine needles the sample solution was filtered through a $0.3 \mu \mathrm{m}$ membrane filter. The residue was separated on a $\mathrm{C}_{18}$ column by high performance liquid chromatography. The mobile phase was $\left(\mathrm{NH}_{4}\right)_{2} \mathrm{HPO}_{4}$ $\mathrm{H}_{3} \mathrm{PO}_{4}$ buffer solution $(\mathrm{pH}=2.7)$. For detection a UV detector at $210 \mathrm{~nm}$ was used. External standard method was performed for the determination of total triterpene content. The test was completed in the Environment Laboratory of Scistd Testing Qingdao Branch, China.

\section{Data processing}

Excel 2003 and SPSS 17.0 Chinese version were used for processing, and one-way analysis of variance was performed.

\section{Results}

\section{Effects of 4 kinds of treatment on the Chl. a content in 3 kinds of seedlings}

Groups of $P$. thunbergii seedlings for Chl. a content test: clean water control group> $P$. thunbergii needle water leaching solution treatment group $>P$. tabuliformis needle water leaching solution treatment group $>P$. koraiensis needle water leaching solution treatment group. $P$. thunbergii needle water leaching solution treatment group, $P$. tabuliformis needle water leaching solution treatment group and $P$. koraiensis needle water leaching solution treatment group showed significantly lower results than clean water control group $(P<0.01, P<0.01, P<0.01)$, and $P$. koraiensis needle water leaching solution treatment group showed significantly lower results than $P$. thunbergii needle water leaching solution treatment group and $P$. tabuliformis needle water leaching solution treatment group $(P<0.01, P<0.01)$ (Fig. 1A).

Groups of $P$. tabuliformis seedlings for Chl. a content test: clean water control group $>$ P. thunbergii needle water leaching solution treatment group $>$. tabuliformis 
needle water leaching solution treatment group $>P$. koraiensis needle water leaching solution treatment group. $P$. thunbergii needle water leaching solution treatment group, $P$. tabuliformis needle water leaching solution treatment group and P. koraiensis needle water leaching solution treatment group showed significantly lower results than clean water control group $(P<0.05, P<0.01, P<0.01)$, and $P$. koraiensis needle water leaching solution treatment group showed significantly lower results than $P$. thunbergii needle water leaching solution treatment group and $P$. tabuliformis needle water leaching solution treatment group $(P<0.01, P<0.01)$ (Fig. 1A).

Groups of $P$. koraiensis seedlings for Chl. a content test: clean water control group $>$ $P$. thunbergii needle water leaching solution treatment group $>P$. tabuliformis needle water leaching solution treatment group $>P$. koraiensis needle water leaching solution treatment group. $P$. thunbergii needle water leaching solution treatment group, $P$. tabuliformis needle water leaching solution treatment group and P. koraiensis needle water leaching solution treatment group showed significantly lower results than clean water control group $(P<0.05, P<0.01, P<0.01)$, and $P$. koraiensis needle water leaching solution treatment group showed significantly lower results than $P$. thunbergii needle water leaching solution treatment group $(P<0.01, P<0.05)$ and $P$. tabuliformis needle water leaching solution treatment group, $P$. tabuliformis needle water leaching solution treatment group showed significantly lower results than $P$. thunbergii needle water leaching solution treatment group $(P<0.01)$ (Fig. 1A).

\section{Effects of 4 kinds of treatment on the Chl. b content in 3 kinds of seedlings}

Groups of $P$. thunbergii seedlings for Chl. b content test: clean water control group> $P$. thunbergii needle water leaching solution treatment group $>P$. tabuliformis needle water leaching solution treatment group $>$ P. koraiensis needle water leaching solution treatment group. $P$. koraiensis needle water leaching solution treatment group showed significantly lower results than clean water control group, $P$. thunbergii needle water leaching solution treatment group and $P$. tabuliformis needle water leaching solution treatment group $(P<0.01, P<0.01, P<0.05)$ (Fig. 1B).

Groups of $P$. tabuliformis seedlings for Chl. b content test: clean water control group $>P$. thunbergii needle water leaching solution treatment group $>P$. tabuliformis needle water leaching solution treatment group $>P$. koraiensis needle water leaching solution treatment group. $P$. thunbergii needle water leaching solution treatment group, $P$. tabuliformis needle water leaching solution treatment group and P. koraiensis needle water leaching solution treatment group showed significantly lower results than clean water control group $(P<0.01, P<0.01, P<0.01)$, and $P$. koraiensis needle water leaching solution treatment group showed significantly lower results than $P$. thunbergii needle water leaching solution treatment group and $P$. tabuliformis needle water leaching solution treatment group $(P<0.01, P<0.01)$ (Fig. 1B).

Groups of $P$. koraiensis seedlings for Chl. b content test: clean water control group $>$ $P$. thunbergii needle water leaching solution treatment group $>P$. tabuliformis needle water leaching solution treatment group $>P$. koraiensis needle water leaching solution treatment group. $P$. thunbergii needle water leaching solution treatment group, $P$. tabuliformis needle water leaching solution treatment group and $P$. koraiensis needle water leaching solution treatment group showed significantly lower results than clean water control group $(P<0.01, P<0.01, P<0.01)$, and $P$. koraiensis needle water leaching solution treatment group showed significantly lower results than $P$. thunbergii needle water leaching solution treatment group and $P$. tabuliformis needle water 
leaching solution treatment group $(P<0.01, P<0.05), P$. tabuliformis needle water leaching solution treatment group was significantly lower than $P$. thunbergii needle water leaching solution treatment group $(P<0.01)$ (Fig. 1B).
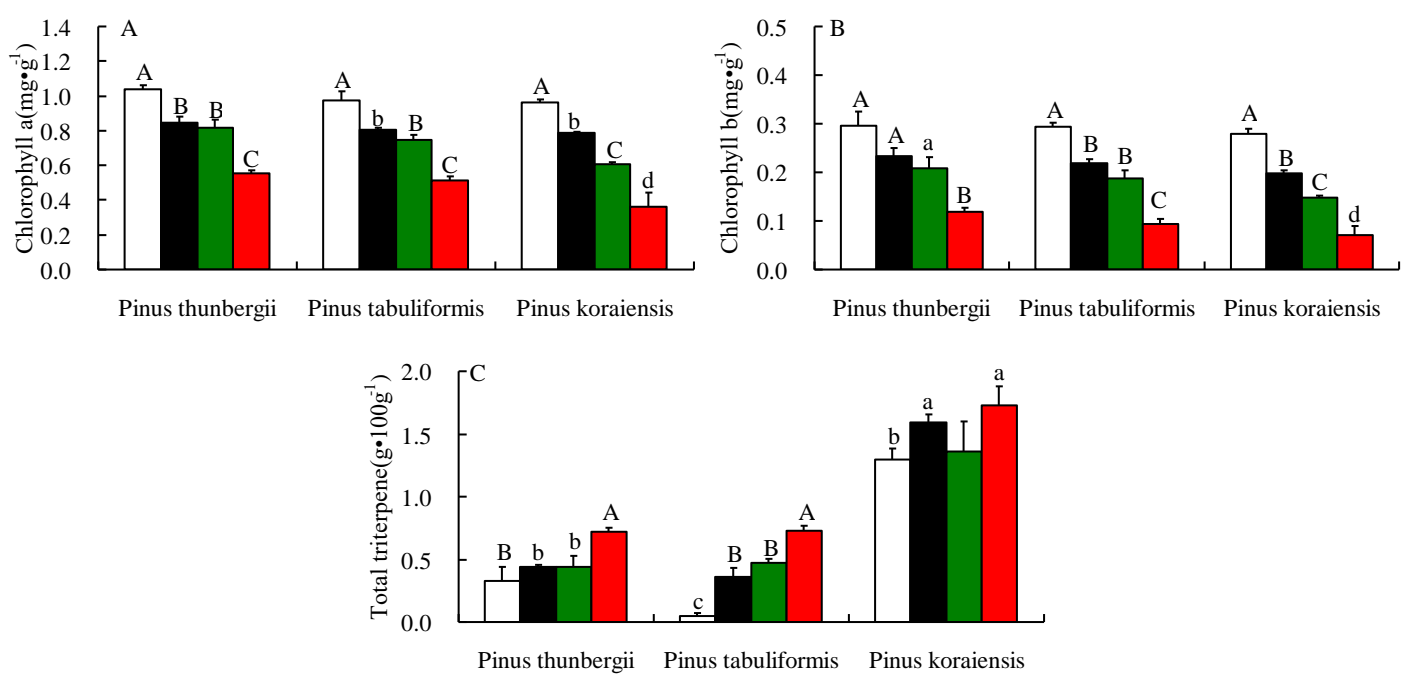

Figure 1. Effects of 4 kinds of treatment on Chl. a (A), Chl. $b(B)$ and total triterpene $(C)$ in 3 kinds of seedlings (average + standard error). $\square$ Clean water control group; $\square$. thunbergii needle water leaching solution treatment group; - P. tabuliformis needle water leaching solution treatment group; - P. koraiensis needle water leaching solution treatment group The difference between the adjacent letters is significant, capitalized letters $P<0.01$, and lowercase letters $P<0.05$

\section{Effects of 4 kinds of treatment on the total triterpene content in 3 kinds of seedlings}

Groups of $P$. thunbergii seedlings for total triterpene content test: P. koraiensis needle water leaching solution treatment group $>P$. thunbergii needle water leaching solution treatment group $>P$. tabuliformis needle water leaching solution treatment group >clean water control group. $P$. koraiensis needle water leaching solution treatment group showed significantly higher results than $P$. thunbergii needle water leaching solution treatment group, $P$. tabuliformis needle water leaching solution treatment group and clean water control group, while $P$. thunbergii needle water leaching solution treatment group, $P$. tabuliformis needle water leaching solution treatment group and clean water control group had no significant difference $(P<0.01, P<0.01, P<0.05)$ (Fig. 1C). P. koraiensis needle water leaching solution had a significant allelopathic effect on $P$. thunbergii seedlings.

Groups of $P$. tabuliformis seedlings for total triterpene content test: P. koraiensis needle water leaching solution treatment group $>P$. tabuliformis needle water leaching solution treatment group $>P$. thunbergii needle water leaching solution treatment group>clean water control group. $P$. koraiensis needle water leaching solution treatment group, $P$. tabuliformis needle water leaching solution treatment group and $P$. thunbergii needle water leaching solution treatment group showed significantly higher results than clean water control group $(P<0.01, P<0.01, P<0.05)$, and $P$. koraiensis needle water leaching solution treatment group showed significantly higher results than $P$. tabuliformis needle water leaching solution treatment group and $P$. thunbergii needle water leaching solution treatment group $(P<0.01, P<0.01)$, while $P$. tabuliformis 
needle water leaching solution treatment group and $P$. thunbergii needle water leaching solution treatment group had no significant difference (Fig. 1C). P. koraiensis needle water leaching solution, $P$. tabuliformis needle water leaching solution and $P$. thunbergii needle water leaching solution had a significant allelopathic effect on $P$. tabuliformis seedlings.

Groups of $P$. koraiensis seedlings for total triterpene content test: P. koraiensis needle water leaching solution treatment group $>P$. thunbergii needle water leaching solution treatment group $>P$. tabuliformis needle water leaching solution treatment group >clean water control group. $P$. koraiensis needle water leaching solution treatment group and $P$. thunbergii needle water leaching solution treatment group showed significantly higher results than clean water control group $(P<0.05, P<0.05)$, while $P$. tabuliformis needle water leaching solution treatment group and clean water control group had no significant difference (Fig. 1C). P. koraiensis needle water leaching solution and $P$. thunbergii needle water leaching solution had a significant allelopathic effect on $P$. koraiensis seedlings.

\section{Relevance of Chl. a, Chl. b and total triterpene}

Analysis indicated significant positive linear correlation between Chl. a and Chl. $\mathrm{b}$ in 3 kinds of seedlings, such as $P$. tabuliformis, $P$. thunbergii and $P$. koraiensis (y $\left.=0.3976 \mathrm{x}-0.1056, R^{2}=0.9435, P<0.01, n=58\right)$ (Fig. 2A). Relevance of Chl. a and total triterpene is significant in 3 kinds of seedlings $\left(y=-0.1303 x^{3}+0.4627 x^{2}-\right.$ $0.4896 \mathrm{x}+0.3312, R^{2}=0.37, P<0.01, n=58$ ) (Fig. 2B). Relevance of Chl. $\mathrm{b}$ and total triterpene is significant in 3 kinds of seedlings $\left(\mathrm{y}=-0.3161 \mathrm{x}^{3}+1.1339 \mathrm{x}^{2}-1.2147 \mathrm{x}+\right.$ 1.0971, $\left.R^{2}=0.3815, P<0.01, n=58\right)$ (Fig. 2C).
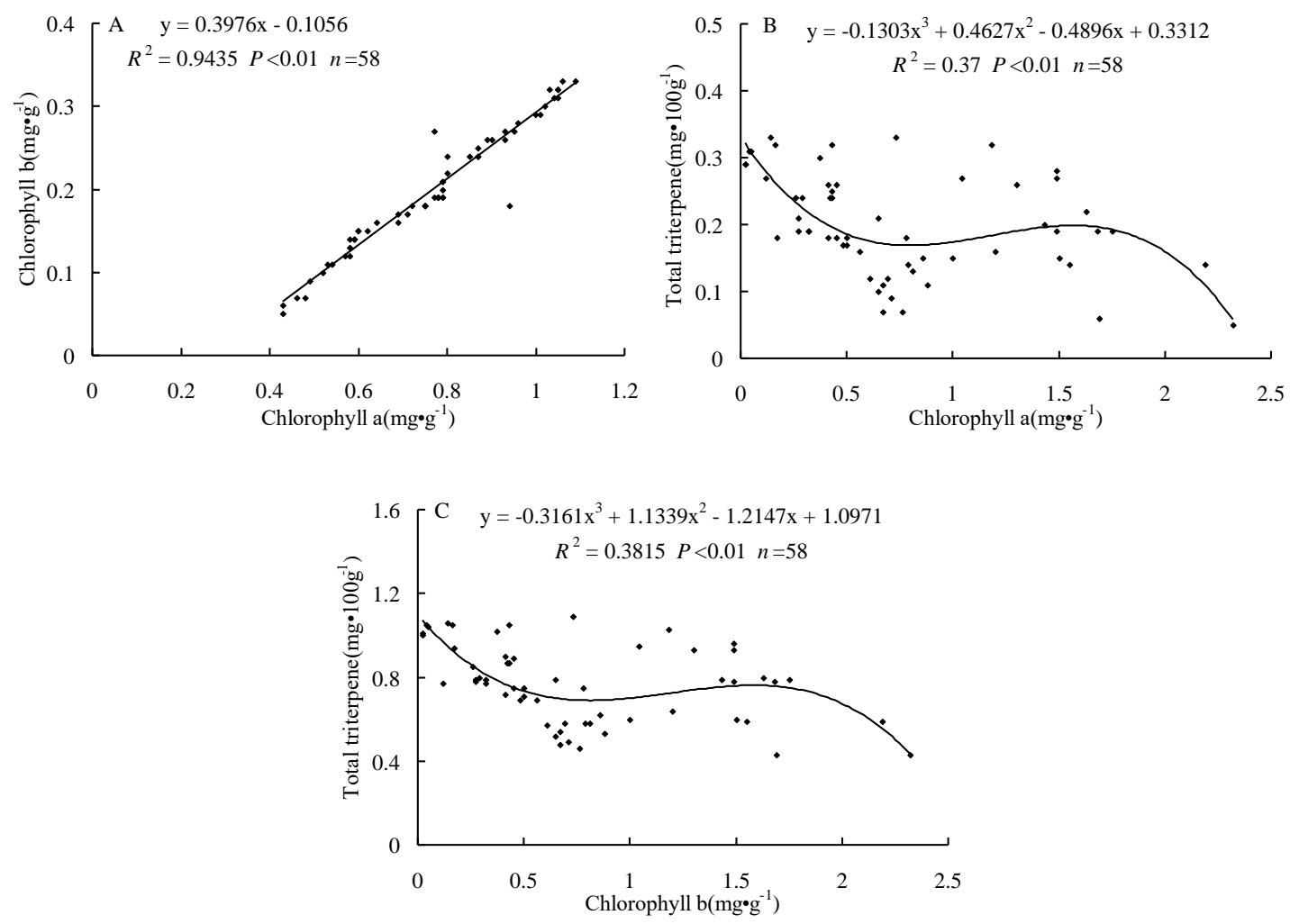

Figure 2. Relevance of Chl. a, Chl. $b$ and total triterpene in 3 kinds of seedlings $(A, B, C)$ 


\section{Discussion}

In recent years, it has been found that allelochemicals can inhibit the absorption and transport of amino acids, and can interfere with the protein synthesis ( $\mathrm{Li}$ et al., 2010), inhibit or damage the synthesis of photosynthetic pigments and accelerate the decomposition of photosynthetic pigments (Poonpaiboonpipat et al., 2013). It also affects the shape and structure of plant cells, and change the transport of nutrients and nutrient accumulation efficiency (Singh et al., 2009; Sunar et al., 2013; Grana et al., 2013), eventually causing a strong change in plant cells and growth of plants. The soil leaching liquor in $P$. koraiensis plantation had suppressive effects on the 7 measured indices, such as germination percentage, germination potential, hypocotyl and radicle length, seedling height, root length and dry mass (Chen et al., 2016). The leaf litter, semi-decomposed litter and surface soil infiltration solution of $P$. tabulaeformis significantly inhibited the growth, chlorophyll content (especially Chl. a content) and net photosynthetic rate of $P$. tabulaeformis seedlings (Jia et al., 2003). Japanese red pine (P. koraiensis) produces resin acids, such as abscisic acid- $\beta$-d-glucopyranosyl ester (Kato-Noguchi et al., 2011), 9 $\alpha, 13 \beta$-epidioxyabeit-8(14)en-18-oic acid and abscisic acid- $\beta$-Dglucopyranosyl ester (Kimura et al., 2015), 15-hydroxy-7-oxodehydroabietate and 7-oxodehydroabietic acid (Kato-Noguchi et al., 2017), which are transported to the surface soil through fallen leaves, inhibiting the growth of roots and shoots of other plants, resulting in sparse understory vegetation. The cedar (Cedrus deodara) leaves volatiles have a strong allelopathic effect, and its main components are terpenoids ( $\mathrm{Li}$ et al., 2015). Their leaf water leaching liquid affects root length, shoot length and chlorophyll content, and reduce seed germination and plant dry weight (Talukdar and Talukdar, 2012; Uddin and Robinson, 2017). Allelochemicals reduce plant absorption and increase the availability of soil nutrients (Aslam et al., 2017; Mohammadkhani and Servati, 2018). Our study showed that pine needle water leaching solutions, such as $P$. tabuliformis, $P$. thunbergii and $P$. koraiensis, significantly inhibit their Chl. a and Chl. $\mathrm{b}$, and will be a norm, which provides a new explanation for the decline in the coniferous forests of northern China. We propose to carry on research in this direction, such as dividing the concentration of the pine needle litter water extract, separating and identifying more new allelochemicals, thus revealing the plant physiological and biochemical reactions and countermeasures of the three major Pinus species in northern China.

\section{Conclusions}

$P$. thunbergii needle water leaching solution treatment significantly reduced chl. a and chl. b contents in $P$. thunbergii seedlings. It greatly reduced the total triterpene content in $P$. tabuliformis seedlings while enhanced the total triterpene content in $P$. tabuliformis. In addition, it significantly reduced the total triterpene content in $P$. koraiensis seedlings while enhanced the total triterpene content in $P$. koraiensis seedlings.

$P$. tabuliformis needle water leaching solution treatment significantly reduced Chl. a and Chl. $\mathrm{b}$ contents in $P$. thunbergii seedlings and Chl. a and Chl. $\mathrm{b}$ contents in $P$. tabuliformis seedlings as well. However, it strengthened the total triterpene content in $P$. tabuliformis seedlings, with the decrease of Chl. a and Chl. b contents in P. koraiensis seedlings. 
Although $P$. koraiensis needle water leaching solution treatment significantly reduced Chl. a and Chl. b contents in $P$. thunbergii seedlings, it dramatically increased total triterpene content in $P$. thunbergii seedlings. And it also caused the total triterpene content in $P$. tabuliformis seedlings to decrease and the total triterpene content in $P$. tabuliformis seedlings to increase. Moreover, it decreased the total triterpene content in $P$. koraiensis seedlings while it increased the total triterpene content in P. koraiensis seedlings.

Comprehensive assessment showed that $P$. thunbergii needle litter water leaching solution had a significantly low inhibition on $P$. thunbergii while a significantly strong inhibition on $P$. tabuliformis and $P$. koraiensis. It also showed a significantly low inhibition on $P$. thunbergii, a moderate inhibition on $P$. tabuliformis and a significantly strong inhibition on $P$. koraiensis. $P$. koraiensis needle litter water leaching solution had a significantly strong inhibition on $P$. thunbergii, $P$. tabuliformis and $P$. koraiensis.

Acknowledgements. Funded by the Fund of Shandong Provincial Key Laboratory of Water and Soil Conservation and Environmental Protection, Linyi University, NO. STKF201603.

\section{REFERENCES}

[1] Albuquerque, M. B. D., Santos, R. C. D., Lima, L. M., Câmara, C. A. G. D., Ramos, A. D. R. (2011): Allelopathy, an alternative tool to improve cropping systems. - Agron Sustain Dev 31: 379-395.

[2] Aslam, F., Khaliq, A., Matloob, A., Tanveer, A., Hussain, S., Zahir, Z. A. (2017): Allelopathy in agro-ecosystems: a critical review of wheat allelopathy-concepts and implications. - Chemoecology 27: 1-24.

[3] Chen, L., Zhang, M., Xin, M., Li, J. D. (2015): Effects of exogenous phenolic acids on allelopathy of potted soybean seedlings. - Agr Sci Tech 16: 1151.

[4] Chen, L. C., Wang, S. L., Chen, C. Y. (2003): Degradation mechanism of Chinese fir plantation. - Chin J Appl Ecol 15: 1953-1957 [in Chinese].

[5] Chen, L. X., Li, S. B., Qiao, L., Bu, F., Duan, W. B. (2016): Influence of leaf litter and soil leaching liquor on seed germination and seedling growth of Pinus koraiensis. Journal of Nanjing Forestry University (Natural Science) 40(2): 81-87 [in Chinese].

[6] Cheng, F., Cheng, Z. H. (2015): Research progress on the use of plant allelopathy in agriculture and the physiological and ecological mechanisms of allelopathy. - Front Plant Sci 1020.

[7] Graña, E., Sotelo, T., Díaz-Tielas, C., Araniti, F., Krasuska, U., Bogatek, R., Reigosa, M. J., Sánchez-Moreiras, A. M. (2013): Citral induces auxin and ethylene-mediated malformations and arrests cell division in Arabidopsis thaliana roots. - J Chem Ecol 39: 271-282.

[8] Jia, L. M., Zhai, M. P., Feng, C. H. (2003): Effects of allelopathic substances on the growth and photosynthesis of Pinus tabulaeformis Seedlings. - J Beijing Fore Univ 25(4): 6-10 [in Chinese].

[9] Kato-Noguchi, H., Fushimi, Y., Tanaka, Y., Teruya, T., Suenaga, K. (2011): Allelopathy of red pine: isolation and identification of an allelopathic substance in red pine needles. Plant Growth Regul 65: 299-304.

[10] Kato-Noguchi, H., Kimura, F., Ohno, O., Suenaga, K. (2017): Involvement of allelopathy in inhibition of understory growth in red pine forests. - J Plant Biol 218: 66-73.

[11] Kil, B. S., Yang, J. Y. (1983): Allelopathic effects of Pinus densiflora on undergrowth of red pine forest. - J Chem Ecol 9: 1135-1151. 
[12] Kimura, F., Sato, M., Kato-Noguchi, H. (2015): Allelopathy of pine litter: delivery of allelopathic substances into forest floor. - J Plant Biol 58: 61-67.

[13] Kong, C. H. (2002): Frontier fields of plant chemical ecology in the 21st century. - Chin J Appl Ecol 13: 349-353 [in Chinese].

[14] Li, D. W., Wang, D. M., Yao, W. X. (2010): Autotoxicity of Pinus tabulaeformisand its ecology significance. - Sci Silva Sin 46(11): 174-178 [in Chinese].

[15] Li, X. F., Jiao, H., Yuan, Y., Tian, S. N. (2015): Allelopathic effects and chemincal components of the volatiles from Cedrus deodara leaves and branches. - Ecol Environ 24: 263-269.

[16] Li, Z. H., Wang, Q., Ruan, X., Pan, C. D., Jiang, D. A. (2010): Phenolics and plant allelopathy. - Molecules 15: 8933-8952.

[17] Mohammadkhani, N., Servati, M. (2018): Nutrient concentration in wheat and soil under allelopathy treatments. - J Plant Res 131: 143-155.

[18] Pan, C. D., Wang, Q., Ruan, X., Li, Z. H. (2009): Biological activity and quantification of potential autotoxins from the leaves of Picea Schrenkiana. - Chin J Plant Ecol 33: 183196 [in Chinese].

[19] Peng, S. L., Shao, H. (2001): Research significance and foreground of allelopathy. - Chin J Appl Ecol 12: 780-785 [in Chinese].

[20] Poonpaiboonpipat, T., Pangnakorn, U., Suvunnamek, U., Teerarak, M., Charoenying, P., Laosinwattana, C. (2013): Phytotoxic effects of essential oil from Cymbopogon citratus and its physiological mechanisms on barnyardgrass (Echinochloa crus-galli). - Ind Crop Prod 41: 403-407.

[21] Singh, A., Singh, D., Singh, N. B. (2009): Allelochemical stress produced by aqueous leachate of Nicotiana plumbaginifolia Viv. - Plant Growth Regul 58: 163-171.

[22] Sunar, S., Yildirim, N., Aksakal, O., Agar, G. (2013): Determination of the genotoxic effects of Convolvulus arvensis extracts on corn (Zea mays L.) seeds. - Toxicol Ind Health 29: 449-459.

[23] Talukdar, D., Talukdar, T. (2012): Alien invasive legumes and allelopathy: a case study at Gangetic West Bengal, India. - Int J Energ Res 4(4): 32-40.

[24] Uddin, M. N., Robinson, R. W. (2017): Allelopathy and resource competition: the effects of Phragmites australis invasion in plant communities. - Bot Stud 58: 29-40.

[25] Wang, Q., Ruan, X., Li, Z. H., Pan, C. D. (2007): Autotoxicity of plants and research of coniferous forest autotoxicity. - Sci Sin 43(6): 134-142 [in Chinese].

[26] Wang, X., Wei, T. X., Zhu, J. Z., Zhao, X. K., Liu, H. Y. (2015): Allelopathic effect of Pinus tabulaeformis root in on loess hilly area. - J Beijing Fore Univ 37(4): $82-89$ [in Chinese].

[27] Wei, J. J., Gao, Y., Zhao, W. G., Liu, J. (2017): Hillside topographic pattern of shrub and herb diversity of forest in Mount Tai of China. - J Environ Prot Ecol 18: 571-580.

[28] Yan, F., Yang, Z. M., Han, L. M. (2000): Review on research methods for allelopathy and allelochemicals in plants. - Acta Ecol Sin 20: 692-696 [in Chinese].

[29] Yin, X. D., Gao, Y., Liu, J., Zhao, W. G. (2018): influence of scenic road corridor to plant diversity in Kunyu Mountain, China. - Appl Ecol Env Res 16: 1461-1469.

[30] Zhang, J., Wang, G. M., Qu, Z. Q., Yang, L., Wang, L. H., Sun, J. G., Zhang, X. N., Xing, H. J. (2012): Preliminary study on allelopathy of Pinus thunbergii. - Shandong Agric Sci 44(7): 37-40 [in Chinese].

[31] Zhou, K., Guo, W. M., Xu, Y. C. (2004): Advances of research on allelopathic potential in compositae. - Acta Ecol Sin 24: 1776-1784 [in Chinese].

[32] Zhu, B. C., Liu, Z. W., Huang, L. J., Bing, Y. H., Zhang, X. X., Lv, C. (2014): Allelopathic effects of humus soil from Pinus tabulaeformis forest on 10 plant species in the loess plateau. - Acta Agrect Sin 22: 1014-1020 [in Chinese]. 